\title{
AERIAL RADIATION DETECTION
}

\author{
September 1999
}




\section{DISCLAIMER}

This report was prepared as an account of work sponsored by an agency of the United States Government. Neither the United States Government nor any agency thereof, nor any of their employees, makes any warranty, expressed or implied, or assumes any legal liability or responsibility for the accuracy, completeness, or usefulness of any information, apparatus, product, or process disclosed, or represents that its use would not infringe privately-owned rights. Reference herein to any specific commercial product, process, or service by trade name, trademark, manufacturer, or otherwise, does not necessarily constitute or imply its endorsement, recommendation, or favoring by the United States Government or any agency thereof. The views and opinions of authors expressed herein do not necessarily state or reflect those of the United States Government or any agency thereof.

Available to the public from:
U.S. Department of Commerce
National Technical Information Service
5285 Port Royal Road
Springfield, VA 22161
(703) 487-4650

Available electronically at http://www.doe.gov/bridge. Available to U.S. Department of Energy and its contractors in paper from:

U.S. Department of Energy

Office of Scientific and Technical Information

P.O. Box 62

Oak Ridge, TN 37831-0062

(423) 576-8401 
DOE/NV11718--242

\title{
AERIAL RADIATION DETECTION
}

\author{
William Quam
}

September 1999

This report is unclassified:

\author{
Authorized Derivative Classifier
}

Work performed under U. S. Department of Energy Contract No. DE-AC08-96NV11718 


\section{TABLE OF CONTENTS}

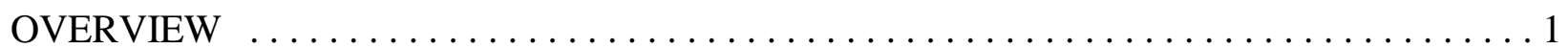

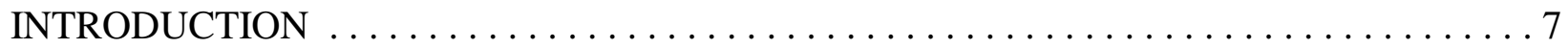

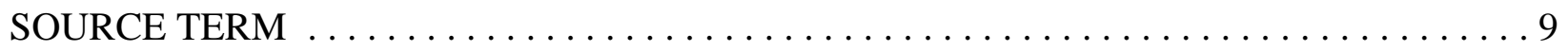

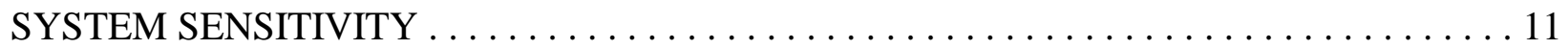

General

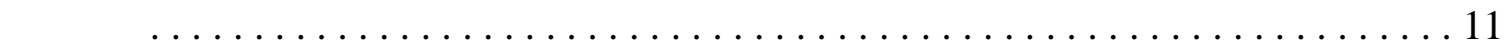

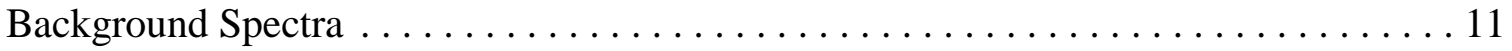

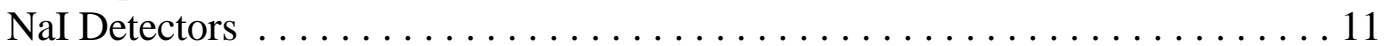

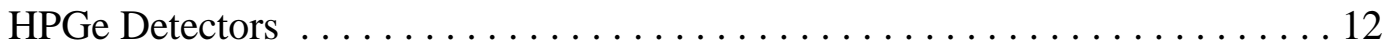

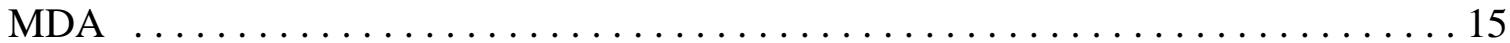

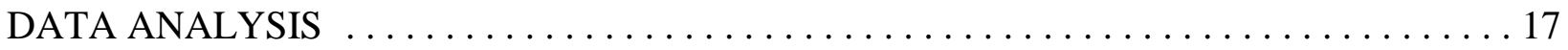

Near Real Time in the Air . . . . . . . . . . . . . . . . . . . . 17

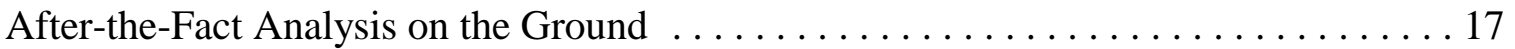

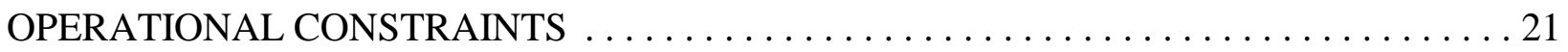

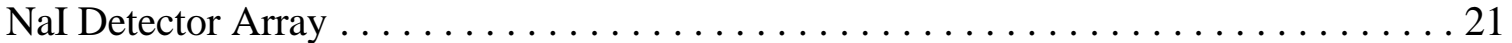

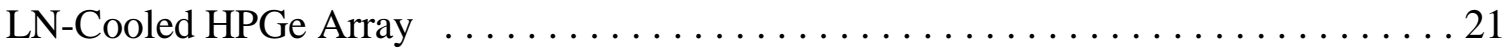

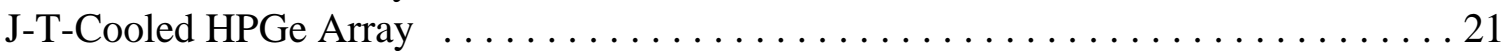

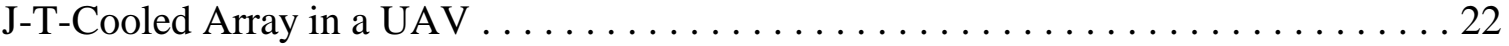

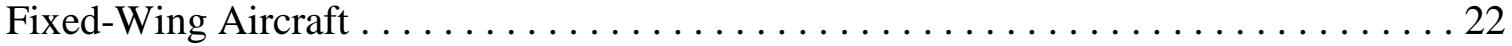

External Stores $v s$ Internal Mounting of Detector Array $\ldots \ldots \ldots \ldots \ldots \ldots \ldots 22$

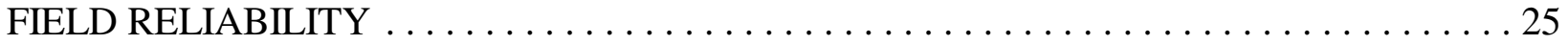

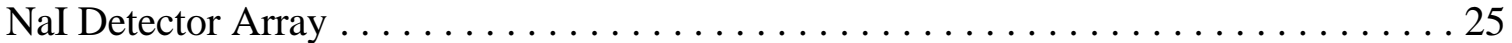

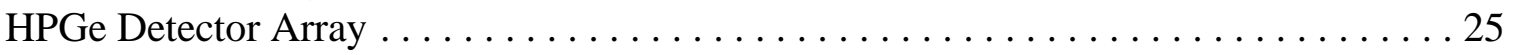

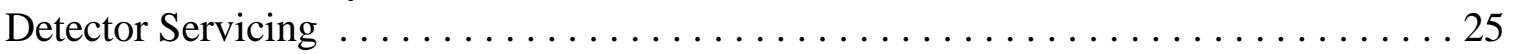

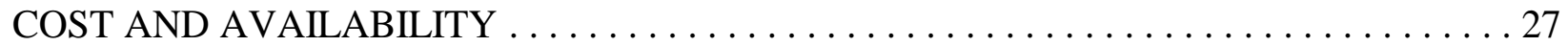

FIELD DETECTION DISCUSSION . . . . . . . . . . . . . . . . . . . . 29

A Large Search Area Requires a Long Time . . . . . . . . . . . . . . . . . . . . . . . 29

Deliberate Shielding of Sources . . . . . . . . . . . . . . . . . . . . . . . . 29

Identification of Sources and Source Producing Operations . . . . . . . . . . . . . 29 


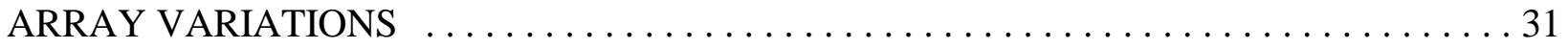

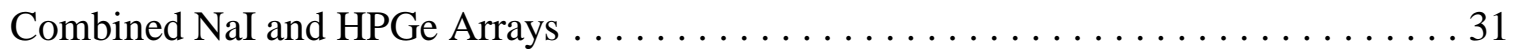

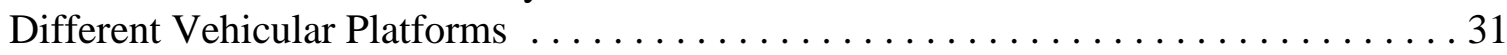

Neutron Detectors Combined with Gamma Detectors $\ldots \ldots \ldots \ldots \ldots \ldots \ldots \ldots$

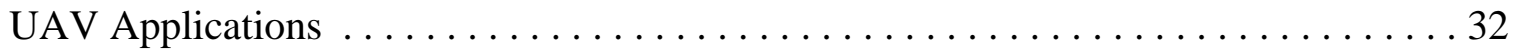

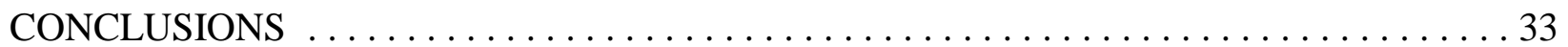




\section{LIST OF FIGURES}

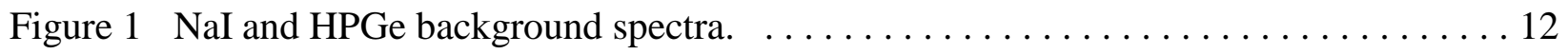

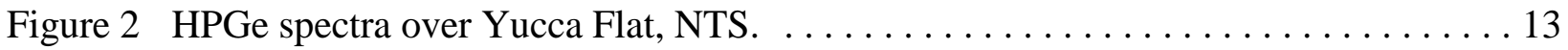

Figure 3 HPGe background spectra near Santa Barbara, CA. . . . . . . . . . . . . . . 14

\section{LIST OF TABLES}

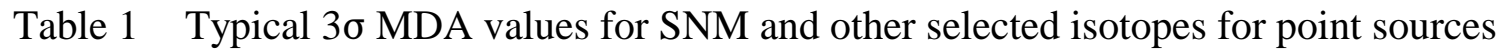
on the soil surface at a 50-foot altitude, and a 1-knot helicopter speed . . . . . . 1

Table 2 MDA, point source on soil surface, 50 foot altitude $\ldots \ldots \ldots \ldots \ldots \ldots \ldots \ldots$

Table 3 Aerial gamma detection systems comparison $\ldots \ldots \ldots \ldots \ldots \ldots \ldots \ldots \ldots$ 


\section{OVERVIEW}

An airborne system designed for the detection of radioactive sources on the soil surface from an aircraft normally senses gamma rays emitted by the source. Gamma rays have the longest path length (least attenuation) through the air of any of the common radioactive emissions and will thus permit source detection at large distances. A secondary benefit from gamma ray detection is that nearly all radioactive isotopes can be identified by the spectrum of gammas emitted. Major gaseous emissions from fuel reprocessing plants emit gammas that may be detected and identified. Some types of special nuclear material (SNM) also emit neutrons which are also useful for detection at a distance.

A gamma ray detection system must be sensitive enough to allow rapid source location from a reasonable altitude with the expectation that sources of small activity will be found. Once a source is found, the system should be able to identify the source isotope by its characteristic gamma emission spectrum. The twin goals of high sensitivity (necessary to find sources) and very good energy resolution (for source identification) are difficult to achieve simultaneously with current detector technology. This note gathers together data describing two types of gamma detector arrays that have been used successfully for this task. The systems will be primarily compared based upon their sensitivity expressed as minimum detectable activity (MDA) and energy resolution. Other system parameters such as, system size and weight, relative costs, etc. are also presented.

One of the most important overall descriptive parameters of a detection system is the MDA. If the system cannot differentiate a source from background, then other operations with the system are pointless. The table below presents a comparison between two systems that have been successfully deployed many times. The data have been derived from experimental airborne measurements.

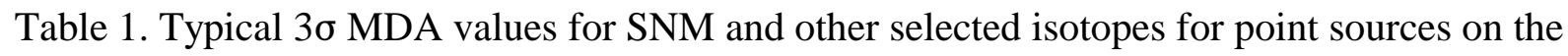
soil surface at a 50-foot altitude, and a 1-knot helicopter speed

\begin{tabular}{ccc} 
Source & $\begin{array}{c}\text { Eight individual } \\
2 \times 4 \times 16 \mathrm{NaI} \text { logs }\end{array}$ & $\begin{array}{c}\text { Twenty individual } \\
65 \% \mathrm{HPGe} \text { detectors }\end{array}$ \\
\hline \hline${ }^{235} \mathrm{U}, 185.72 \mathrm{keV}$ & $47 \mathrm{~g}, 100 \mu \mathrm{Ci}$ & $29 \mathrm{~g}, 62 \mu \mathrm{Ci}$ \\
${ }^{239} \mathrm{Pu}, 375.92 \mathrm{keV}$ & $0.8 \mathrm{~g}, 48 \mathrm{mCi}$ & $0.2 \mathrm{~g}, 14 \mathrm{mCi}$ \\
${ }^{241} \mathrm{Am}, 59.35 \mathrm{keV}$ & $100 \mu \mathrm{Ci}$ & $44 \mu \mathrm{Ci}$ \\
${ }^{137} \mathrm{Cs}, 661.65 \mathrm{keV}$ & $90 \mu \mathrm{Ci}$ & $28 \mu \mathrm{Ci}$
\end{tabular}

The 2 x 4 x 16-inch sodium iodide (NaI) scintillators represent an older technology that has been in use on both fixed wing aircraft and helicopters. These systems are very sensitive, but have poor energy resolution leading to the high MDA values given. This is due to the significantly larger background counts found in the wide energy window around each gamma line. The NaI 
system has a resolution of about $10 \%$ full width half maximum (FWHM) at the $186 \mathrm{keV}$ line from ${ }^{235} \mathrm{U}$ and an energy window is taken as twice the FWHM.

The high purity Germanium (HPGe) system has about $10 \%$ of the peak counting rate of the NaI system, but has an energy resolution of about $1 \%$ at the $186 \mathrm{keV}$ line. This leads directly to a smaller MDA.

The tabular data above were determined for a slow speed helicopter (approximately 1 knot) flying directly over a point source. Increasing the aircraft speed has only a small effect until the effective footprint of the detector system (about 100 feet in diameter at a 50-foot altitude) is transited in 1 second, the usual data collection time. This is about 60 knots. Increasing the speed beyond this value will increase the MDA approximately linearly with speed since less time over the source will yield fewer source counts compared to background which is constant. Increasing the altitude, say to 100 feet, will increase the MDA by a factor of five for ${ }^{235} \mathrm{U}$.

A fixed wing aircraft can safely fly at 200 feet or greater. The effective footprint is about 400 feet in diameter at this altitude implying a speed of 240 knots for a 1 second transit over the footprint. This will theoretically allow collection of approximately the same number of gammas from the source as the 50-foot helicopter flight, but air attenuation will reduce the actual number arriving at the detector. The result is a factor of approximately 30 increase in the MDA for ${ }^{235} \mathrm{U}$ compared to the 50-foot altitude. If safety considerations require a higher aircraft speed (say 300 knots), the MDA will be increased more.

If the aircraft ground track misses the source by a 50-foot offset at an altitude of 50 feet, the MDA will increase by a factor of approximately six. This is due to the smaller effective area of the detector array when viewed from an angle other than directly below. As the offset distance increases, the effective detector area decreases due to geometric effects and the MDA increases further. There will also be an additional $1 / \mathrm{r}^{2}$ decrease and additional air attenuation since the path length is longer. The same geometric factor also applies to the fixed-wing aircraft at a 200-foot altitude and a 200-foot offset.

These considerations indicate that accurate recording of flight data, such as altitude and geographical position along with the gamma data, is an important feature of the airborne data acquisition system. Both the NaI and the HPGe systems produce large amounts of data. About 600 Mbytes per 2 hours of flying time is a reasonable estimate for the HPGe system. High-speed data handling electronics is needed in the aircraft.

Data analysis is broadly similar for both technologies and must be done rapidly if the deployment is to take advantage of newly found data. The NaI systems with their poor resolution have presented many problems in identification of the actual isotopes contributing to the detector spectrum. The best results are obtained by employing a combination of computer data reduction together with a trained analyst. The HPGe systems with very good energy resolution may enjoy nearly automatic data analysis and source isotope identification. In this instance, the huge amount of data generated will impede rapid post-flight analysis unless the data can be easily 
transferred to the land-based analysis system. Removable hard discs have been used successfully for this purpose.

Real-time data processing on board the aircraft is possible, but usually is limited by available aircraft power, the additional weight of more complex processing equipment, and, most of all, by the ability of the airborne equipment operator to interpret the data and to act upon it. In many instances, with $\mathrm{NaI}$ detectors it was found that simple count-rate increases were the best indicator of source presence. The HPGe systems have been provided with various energy windows that permit display of isotope related counting rates. This may be a suitable display scheme.

The NaI scintillator technology has been in use for years. The detector cost is fairly low, approximately $\$ 6,000$ per detector, but the electronics must be custom designed to handle all the parameters that must be recorded. The overall system weight is low, approximately 200 pounds for the eight-detector array and 50 pounds for modern electronics for a total of 300 pounds, and will fit on a small helicopter such as a BO-105.

Large airborne arrays of HPGe detectors are a relatively new concept. The cost is considerably greater than a $\mathrm{NaI}$ array, ranging from $\$ 40,000$ to $\$ 80,000$ per detector depending upon the detector efficiency ( 65 to $110 \%$ ). The electronics will be custom designed for the application (see below).

The system whose data are given in the table above was built in 1989. It weighed nearly 1800 pounds and required a large helicopter for deployment. It also used liquid nitrogen (LN) as the cryogen necessary for detector operation. This introduces an operational requirement of LN for field support. At times this has been a problem since LN cannot be stored for any length of time, pending use in the system.

Recent developments using Joule-Thomson (J-T) cooling in place of the LN have shown that comparable systems can be constructed that have as good or better energy resolution, better sensitivity (110\% detectors versus $65 \%$ detectors), and lighter, overall weight (760 pounds compared to nearly 1800 pounds for the LN technology). This will enable a 20-detector J-T-cooled array to be carried on reasonably small helicopters. In addition the high pressure argon gas needed for the J-T coolers can be stored indefinitely and is much less of a problem in the field. One system using $12 \mathrm{~J}$-T-cooled HPGe detectors (all that were available at the time) has been assembled and was flown successfully in 1996.

System electronics for HPGe arrays will be a custom design. VME hardware was used on both the LN and J-T-cooled systems to sustain high-data transfer rates. Each of the 20 detectors had it's own 16,000-channel analog-to-digital converter, high voltage power supply, amplifier, etc. Two 68000 series processors were used to handle the data streams. Providing near real-time data display for the airborne operator requires another processor. Global positioning systems (GPS) location signals used another dedicated processor. Newer, faster, PC-based systems may simplify some of this complexity. 
The usual aircraft of choice for gamma survey work is a helicopter. The main reason is that the helicopter can fly low and slow, both of which are an advantage for data collection. A fixed-wing aircraft might be able to fly safely only at 200 feet and above. The MDA is increased by a factor of 30 or more for the fixed-wing scenario. In addition, the fixed-wing aircraft speed may be as high as 300 knots, further increasing the MDA.

Both the fixed-wing and helicopter aircraft generate vibration-induced noise in the detection systems. Mechanical mounting of the detectors must include vibration mitigation hardware for successful operation. The detector systems are usually contained in an external pod carried on ordnance hangers. This is convenient from a physics standpoint since it minimizes attenuation of source gammas by aircraft hardware. However, it may be necessary to obtain an airworthiness certificate for an external store in order to meet safety requirements. This can be an expensive and time consuming task. Some helicopters have only a minimum amount of material under the floor (no fuel in particular). If such a helicopter can be used, it may be possible to mount the detector inside with greatly simplified safety requirements. Even so the low energy gamma response, for ${ }^{241} \mathrm{Am}$ for example, will suffer.

Experience has shown that $\mathrm{NaI}$ systems are reliable in the field. In addition, if a detector fails, it is very easy to replace. The LN-cooled HPGe systems are also reliable, but there are more things to go wrong. The $\mathrm{LN}$ is retained in a vacuum-insulated, metal thermos bottle (called a Dewar) that is coupled to the detector (the germanium crystal) allowing the germanium to be cooled to $77 \mathrm{~K}$, the boiling point of LN. The detector array is made up of 10 individual HPGe detectors on one common Dewar. The HPGe detector system has two of these arrays. If a detector loses its vacuum insulation on a common-Dewar array, the whole Dewar may fail reducing the data acquisition capabilities dramatically. Replacement of a detector in the field is very difficult. The J-T-cooled array does not have a common Dewar (or any Dewar for that matter) and individual detectors may be more easily replaced or removed from the system.

It may be possible to combine parts of the NaI system with a small HPGe array to advantage. For example the NaI array is used to detect a source anomaly. The helicopter is then slowed to a hover or flown at a much lower speed over the anomaly long enough that the HPGe array can gather sufficient data for isotope identification. Landing might also be an option and, if possible, could allow use of a single large HPGe detector close to the source.

Urban areas with many buildings, complicated street systems, power lines, etc. are difficult terrain to search, even with a helicopter. In these cases, it is better to mount the detector system on a vehicle and drive. All the detector systems noted above have been used in vehicles with good results. For example, this search process places the detectors closer to the source(s) which will result in a better detection capability. At the same time, the detectors are closer to the soil and pavement surface which increases the background due to natural radioactivity and thus increases the MDA. The background will also tend to appear more variable due to radioactive components of pavement, concrete, and various building materials. This will complicate the analysis process. 
Another detection method that is possible is use of an unoccupied aerial vehicle (UAV) as the aerial platform for a light weight, J-T-cooled detector. A UAV can be safely flown close to the ground surface resulting in a large increase in gamma counts, about a factor of 25 for 10 feet compared to 50 feet for a helicopter with a larger array. Nearly the same detection sensitivity is possible with one large HPGe detector as can be obtained with a larger array. This is an unexplored area of technology at present.

There are other important considerations in searching for sources. A knowledgeable individual can readily hide the SNM by enclosing it in an inch of lead, burying it underground a few feet or otherwise shielding it. In these instances, detection of the sources by NaI or HPGe gamma systems may be impossible. These shielding methods will have little, if any, effect on neutron emission and it may be possible to use neutron detector arrays to find plutonium isotopes. Large ${ }^{3} \mathrm{He}$ neutron detector arrays made up of eight or more polyethylene moderated counter tubes are a suitable system. 
This page intentionally left blank 


\section{INTRODUCTION}

Aerial surveying for gamma sources consists of searching for the source(s) and then identifying the isotope. Search requires a high sensitivity detection system to provide a sufficiently large counting rate that small variations in the natural background can be readily separated from a source-caused anomaly. Isotope identification needs a detection system with good gammaenergy resolution to enable separation of source gamma lines from those of the ubiquitous gamma background from naturally occurring uranium, thorium, and potassium. Fallout from past weapon tests, reactor accidents, or radiation spills must also be discriminated against. Lastly, it must be possible to determine the emission rate of the isotope to assess the importance of the find.

Typical aerial platforms used for gamma survey work are small, slow, fixed-wing aircraft or helicopters. A search will typically deploy the gamma detection equipment at 50 or 100 feet above the local terrain. Flight lines are arranged to be 1 to 3 altitude widths apart, i.e., 50 to 150 feet apart for a nominal 50-foot altitude, which allows rapid coverage with minimal chance of missing a weak source. Precise flight navigation is required and may be difficult in varying field conditions. Gamma spectral data are collected and referenced to a geographical location during flight. Analysis of the radiation data may be provided roughly in real-time with more thorough processing performed at a post-mission ground site.

There are two types of gamma detectors suitable for aerial survey work: large $\mathrm{NaI}(\mathrm{Tl})$ scintillators (typically $2 \times 4 \times 16$ inches) which provide very high sensitivity or large HPGe detectors which offer very good energy resolution. Either of these detector types are available commercially. The NaI scintillators are typically employed in arrays of 8 to 12 units contained in a pod or housing. The HPGe detectors have been used in arrays of 20 detectors also contained in pod or housing. In both systems the pod has been mounted as an external store, usually on a helicopter. However, some helicopters that do not have fuel tanks under the floor may use either detector type as an internal payload. If this is possible the airworthiness certifications are greatly simplified.

These detection systems collect considerable amounts of data, more for the high resolution HPGe systems than for the NaI scintillators. For example, a 20-detector HPGe system will produce 600 Mbytes of data for a 2-hour flight. Special purpose electronics is required for the multiple detectors and the large data rates encountered. Usually the electronics are specifically designed for the system. Data analysis techniques are unique and difficult due to the large amount of data. Specialized software is required to enable timely processing of the data to provide the location and identity of gamma emitting isotopes.

Field deployment has some similarities between the two types of detection systems, but there are decisive differences in the required logistics. Clearly an aircraft is required as a platform for either system and is similar for the NaI and the HPGe. The NaI detectors need little, if any, field service other than routine energy calibration. However, the HPGe detectors will require cryogen (LN) replenishment every 4 hours or so during field operations for a large array of older technology detectors. LN may be a problem to obtain in some forward areas since it cannot be 
stored for more than a few weeks even with the best technology. Some training is required of support personnel for LN handling to avoid personal hazard and possible damage to the equipment.

A newer cooling technology using the J-T effect and high-pressure argon gas needs less direct attention, but the gas supply must be maintained continuously during field operations. The gas can be stored indefinitely and can thus be shipped with the equipment during deployment. The J-T cooling technique produces a lighter-weight detector and array. This result can be used to extend the flying time, or minimize the helicopter size needed for system deployment. A UAV can also be considered as a viable platform for one or two J-T-cooled HPGe detectors.

The sections below describe the two main detector systems with respect to their sensitivity, energy resolution, and field limitations. It is assumed that the necessary electronics, data recording hardware and methods, and suitable software are available for system operation. These components are similar in concept for the two systems, but differ in actual implementation. It is not expected that the electronics are a deciding factor in any system selection. Brief mention will be made of salient differences. 


\section{SOURCE TERM}

This paper is restricted to three sources: ${ }^{235} \mathrm{U}$ in the form of highly enriched uranium (HEU), ${ }^{239} \mathrm{Pu}$ as metal, and ${ }^{137} \mathrm{Cs}$ as a surrogate for non-SNM source material likely to be found in illegal transport. Medical isotopes such as ${ }^{99 \mathrm{~m}} \mathrm{Tc}$ and ${ }^{201} \mathrm{Tl}$ are not covered explicitly; however, the approximate detection sensitivity may be inferred from the data supplied. Note that it is important to be able to distinguish between common medical isotopes and $\mathrm{SNM},{ }^{235} \mathrm{U}$ in particular. A high resolution gamma detector is required.

The gamma lines considered for each of the three isotopes are:

- ${ }^{235} \mathrm{U}-185.72 \mathrm{keV}$, yield is 0.561 gammas per disintegration

- ${ }^{239} \mathrm{Pu}-375.02 \mathrm{keV}$, yield is $1.6 \times 10^{-3}$ gammas per disintegration

- ${ }^{137} \mathrm{Cs}-661.66 \mathrm{keV}$, yield is 0.851 gammas per disintegraton

The following isotope is a decay product of ${ }^{241} \mathrm{Pu}$ and usually accompanies reactor-produced, chemically-separated ${ }^{239} \mathrm{Pu}$. It may be a useful tracer under some circumstances.

- ${ }^{241} \mathrm{Am}-59.53 \mathrm{keV}$, yield is 0.353 gammas per disintegration

Uranium has other gamma lines that may be useful for detection purposes: 143.78 and $205.31 \mathrm{keV}$ are likely candidates. While not considered in this note, these lines can be used for help in field identification.

Plutonium also has other gamma lines between 200 and $450 \mathrm{keV}$ with yields 0.1 to 1.0 of the $375 \mathrm{keV}$ line. These are not explicitly considered in this note, but could be useful for isotope identification (ID) in the field. In addition, ${ }^{240} \mathrm{Pu}$ emits neutrons due to spontaneous fission. Typical reactor-produced plutonium contains significant amounts of ${ }^{240} \mathrm{Pu}$ in addition to the fissionable ${ }^{239} \mathrm{Pu}$, thus neutron emission is part of the plutonium signature. Detection of these particles is not considered in detail in this note, but is a very useful part of an ID scheme.

The physical size of the sources that are at the MDA (see MDA section) is between 30 and 50 grams for ${ }^{235} \mathrm{U}$ (approximately a cube of 1.2 to $1.4 \mathrm{~cm}$ on a side) and 0.2 to 0.8 grams of ${ }^{239} \mathrm{Pu}$ (approximately a cube 0.2 to $0.3 \mathrm{~cm}$ on a side) for a 'point' source at a 50-foot altitude. These data are for unshielded sources and large 8-detector NaI or 20-detector HPGe arrays. Shielding, smaller detectors, or higher altitudes imply larger sources for minimum detectable sizes. 
This page intentionally left blank 


\section{SYSTEM SENSITIVITY}

\section{General}

Detection sensitivity depends upon several factors:

- detector energy resolution,

- background counting rates versus energy,

- system noise (at least at low gamma energies),

- detector altitude above the source(s), and

- $\quad$ source and platform parameters such as source physical size (point versus distributed) and platform speed and spatial offset from the point source.

These are qualitatively related as follows. A radiation source is 'detected' if the counts due to that source exceed the background counts normally found within a narrow energy window. The window width is twice the detector-energy resolution at the gamma energy in question. In this note, the net counts, defined as gross counts minus the background counts in the energy window, must be three times the standard deviation $(3 \sigma)$ of the background in the energy window to qualify as 'detected'. The source activity causing these counts is calculated from the $3 \sigma$ deviation by using energy-dependant efficiency data. This source activity is called the MDA.

System noise will cause a spread in the energy window by degrading the detector's energy resolution which increases the background counts. More source counts will then be required to pass the $3 \sigma$ test of detectability resulting in a higher MDA.

The platform altitude will affect the source counts due to $1 / \mathrm{r}^{2}$ changes. There is significantly increased air attenuation for lower-energy gammas as the altitude increases. Both these altituderelated counting rate changes will increase the MDA as the altitude increases.

Lastly, if the detector platform passes rapidly over a point source, there is less time to integrate gamma counts from the source leading to a reduced likelihood of detection. Similarly, if the platform passes a point source at a large offset distance, the number of counts will also decrease. These latter two parameters suggest a tight spacing between flight lines, and a slow platform velocity is an optimal search technique. In practice this increases the survey time and cost.

\section{Background Spectra}

\section{NaI Detectors}

A background spectrum for an array of eight 2 x 4 x16-inch NaI 'logs' is shown in Figure 1. These data were taken at a test range in Calvert County, Maryland, and have been provided through the courtesy of Dr. R. Maurer. Small corrections have been made for non-terrestrial gammas from the aircraft platform, radon progeny, and cosmic rays. The NaI spectra are broad, relatively smooth, and nearly devoid of peaks. The detector resolution was about 10\% FWHM and individual peaks are $\pm 10 \%$ wide on the energy axis or twice the FWHM. This energy range or window is used to determine the background for MDA calculations. 


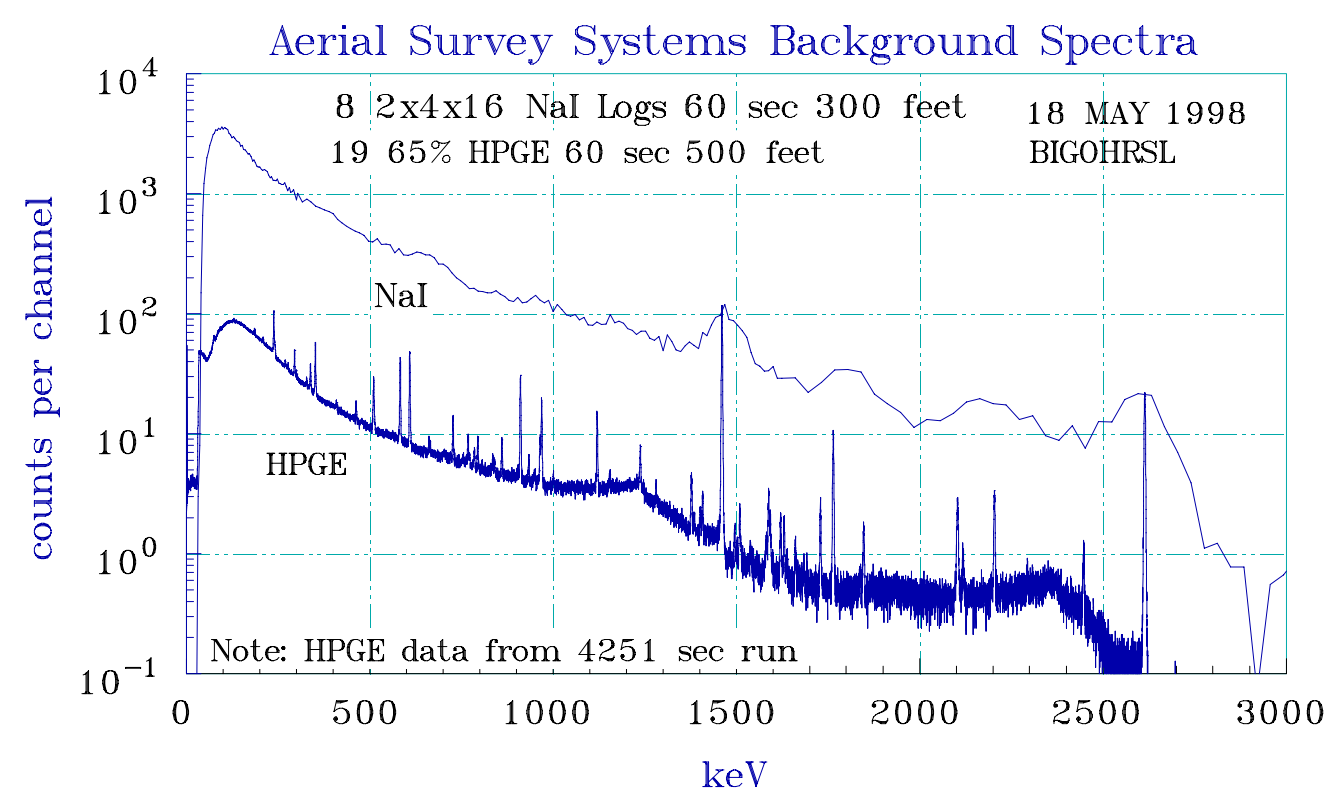

Figure 1. NaI and HPGe background spectra.

\section{HPGe Detectors}

Plotted on the same figure is a spectrum taken with a 19-detector HPGe array in the desert near Las Vegas, Nevada. The spectrum plotted is a direct sum of the individual detector spectra. The individual HPGe detectors had a nominal efficiency of $65 \%$ of a $3 \times 3$-inch NaI detector at $25 \mathrm{~cm}$ from a ${ }^{60} \mathrm{Co}$ source ( $1.332 \mathrm{MeV}$ peak), hence the $65 \%$ designation. The original altitude (500 feet) was comparable to the NaI spectrum, and the collection time has been made equal by dividing the original 4251 second run to yield a 60 -second equivalent.

Two things are immediately apparent: the HPGe spectrum has many sharp peaks, and it is lower in intensity. The HPGe detector array has a resolution varying from $1.3 \mathrm{keV}$ FWHM at $59.35 \mathrm{keV}$ to $3.6 \mathrm{keV}$ FWHM at $2614.35 \mathrm{keV}$. The resolution can be approximately given by: FWHM $=0.4727 \mathrm{keV}^{0.2557}$ over this energy range. The resolution at $185.74 \mathrm{keV}$ is $1.91 \mathrm{keV}$ or $1 \%$ compared to the NaI detector array resolution of $10 \%$. This will mean significantly less background counts under the peak directly leading to a lower MDA.

Other data (not shown here) suggest that an individual 65\% HPGe detector has about 1/20 the peak efficiency for ${ }^{40} \mathrm{~K}$ (at $1460 \mathrm{keV}$ ) of one $2 \times 4 \times 16$-inch NaI log. Thus an array of $2065 \%$ HPGe detectors has about 1/10 the peak counting rate of an 8-detector array of $2 \times 4 \times 16$-inch $\mathrm{NaI}$ logs. In practice, the lack of source-caused counts is partially compensated for by the significantly smaller number of background counts in a peak energy window and the HPGe MDAs are smaller (see below) than those found for the NaI array. 
Two other HPGe background spectra are presented in Figures 2 and 3. Figure 2 was obtained at an altitude of 100 feet over a region called Yucca Flat at the Nevada Test Site (NTS). A portion of the whole spectrum is shown covering the region where ${ }^{235} \mathrm{U}$ and ${ }^{239} \mathrm{Pu}$ have major peaks.

There is a small peak at $185.99 \mathrm{keV}$ that is due to ${ }^{226} \mathrm{Ra}$, part of the ${ }^{238} \mathrm{U}$ decay chain. This will complicate detection of the ${ }^{235} \mathrm{U}$ line at $185.72 \mathrm{keV}$ and may prevent detection of any HEU present. The usual solution to this problem is calculation of the expected ${ }^{226} \mathrm{Ra}$ counts from the ${ }^{214} \mathrm{~Pb}$ peak counts at 295.22 and $351.99 \mathrm{keV}$. Any excess is due to ${ }^{235} \mathrm{U}$. For the HPGe detectors used at altitudes of 50 to 100 feet, the count ratio is approximately 0.51 at $295.22 \mathrm{keV}$ and 0.30 at $351.99 \mathrm{keV}$. This is not a very accurate method and frequently suffers from poor statistics.

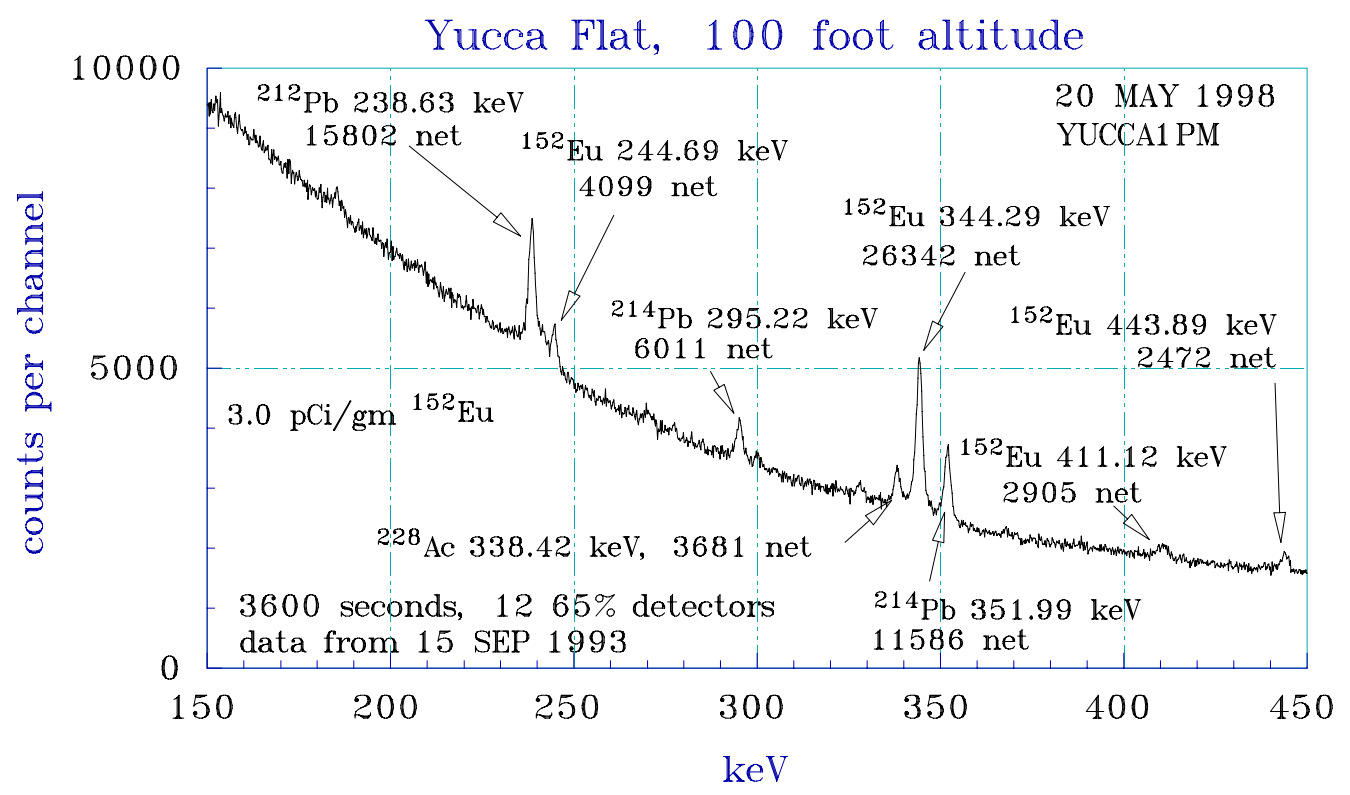

Figure 2. HPGe spectra over Yucca Flat, NTS.

The energy region near $375 \mathrm{keV}$ in the Yucca Flat spectrum (Figure 2) is free of peaks and could be used for ${ }^{239} \mathrm{Pu}$ detection. The ${ }^{152} \mathrm{Eu}$ lines identified are due to neutron activation of natural europium in the NTS soil. The europium soil concentration is estimated to be $3.0 \mathrm{pCi} / \mathrm{g}$. The other lines are natural background from $\mathrm{U}$ and Th daughters.

Figure 3 presents data from a region near Santa Barbara, California, and was taken from a stationary vehicle with the detector array about 5 feet above the soil surface. The majority of the gamma lines seen are from natural $\mathrm{U}$ and $\mathrm{Th}$ as in Figure 2; however, there is a significant ${ }^{226} \mathrm{Ra}$ peak at $185.99 \mathrm{keV}$. The approximate calculation methods noted above suggest an average of 18,500 counts compared to the actual value of 16,290 counts, a difference of $13.5 \%$. Clearly, detection of HEU in this area would have to allow for the presence of ${ }^{226} \mathrm{Ra}$ in the soil. In fact, other ${ }^{235} \mathrm{U}$ peaks (154.18 or $163.37 \mathrm{keV}$ ) may be better suited for HEU detection in this location. 


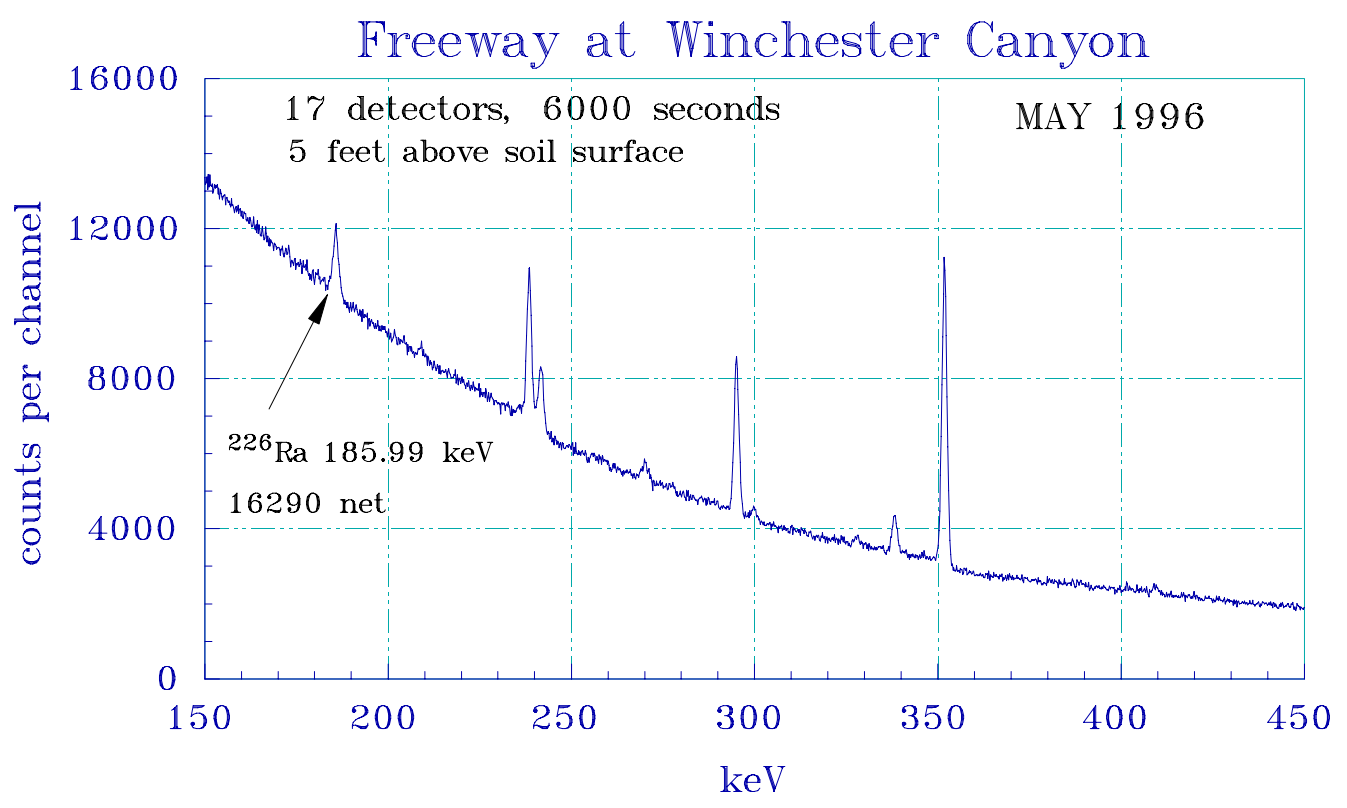

Figure 3. HPGe background spectra near Santa Barbara, CA.

The energy region around $375 \mathrm{keV}$ is again free of background peaks and is suitable for ${ }^{239} \mathrm{Pu}$ detection. Note that there is a small peak near $410 \mathrm{keV}$ that was obscured in the Yucca Flat spectrum by the ${ }^{152} \mathrm{Eu}$ gamma at $411.12 \mathrm{keV}$. Either of these gamma peaks would make analysis of the ${ }^{239} \mathrm{Pu}$ line at $414 \mathrm{keV}$ more complicated.

\section{Summary of Background Spectra Considerations}

$\mathrm{NaI}$ detectors have broad peaks due to natural background gamma emitters. The detector energy resolution is about $\pm 10 \%$. The overall counting rates for $2 \times 4 \times 16$-inch logs are high. The broad energy resolution implies that the net counts in excess of background within an energy region must be large to be detected above the statistical fluctuations.

HPGe detectors have very narrow peaks with an energy resolution of $1 \%$ at the ${ }^{235} \mathrm{U} 185.74 \mathrm{keV}$ line. The overall counting rates for an HPGe array are about 1/10 that of the NaI log array. The narrow energy resolution results in a much smaller number of net counts needed for detection above statistics compared to NaI detectors. As will be shown below, this leads to smaller MDAs for the HPGe arrays.

Various background peaks seen in both the NaI and HPGe spectra must be accounted for in detection and identification of ${ }^{235} \mathrm{U}$ and ${ }^{239} \mathrm{Pu}$ gamma lines. This is made particularly difficult with $\mathrm{NaI}$ detectors because the broad energy resolution will not permit discrimination between many of the source peaks and the natural background peaks. 


\section{MDA}

The MDA in this note is determined from the detector counts in a gamma energy window surrounding the gamma line. The energy window width is taken to be twice the detector energy resolution at FWHM and is approximately $\pm 10 \%$ for NaI detectors. The energy window width was determined separately for each gamma line for the HPGe detectors based upon the measured energy resolution. The source-caused counts within this energy window must exceed three standard deviations $(3 \sigma)$ of the background counts within the same energy window to be 'detected'. This is called the $3 \sigma$ MDA.

Each detector system has a different background rate and a different energy resolution leading to differing $3 \sigma$ requirements. Figures 1 through 3 are representative of the background spectra from the NaI and HPGe systems.

The $3 \sigma$ counts above background within a given energy window may be converted into source activity on the soil surface by correcting for detector intrinsic efficiency, detector surface area, yield of an individual gamma during isotopic decay, pod and air attenuation, and altitude. A generic equation relating these parameters for a point source is:

$$
\frac{\text { net counts in peak }}{\mathrm{sec}}=\frac{\text { Eff } \times \text { detector area } \times \text { source } \gamma^{\prime} \text { s per } \mathrm{sec} \times \text { air and pod transmission }}{4 \times \pi \times(\text { altitude })^{2}}
$$

where Eff is the intrinsic efficiency and has units of $c / \gamma$.

The source $\gamma^{\prime} s$ per second are obtained from:

$$
\text { source } \gamma^{\prime} \text { s per sec }=C i \times \text { yield } \times 3.7 \times 10^{10}
$$

The detection system collects counts throughout the energy spectrum during operation. A background spectrum normally is accumulated over a suitable local area during preliminary operations. This spectrum, similar to Figures 1 through 3, enables a counting rate to be determined for the energy window for each gamma line. This background rate will yield a $3 \sigma$ rate which then fixes the MDA.

In practice the detector system is set to collect a spectrum every second for a typical NaI array, or continuously, with arrival-time-stamps for every gamma detected, for an HPGe array. The analysis software then produces a counting rate within a given energy window for every second of flight time. This counting rate is compared with the $3 \sigma$ background rate in each energy window to determine if a detection has been made.

The MDAs for a very slow pass (nominally 1 knot) over an unshielded point source on the soil surface calculated from the background spectra given above and Equations 1 and 2 are presented in Table 2. 
Table 2. MDA, point source on soil surface, 50 foot altitude

Source $\quad$ Eight individual $\quad$ Twenty Individual

\begin{tabular}{ccc} 
Source & $\begin{array}{c}\text { Eight individual } \\
2 \times 4 \times 16 \mathrm{NaI} \text { logs }\end{array}$ & $\begin{array}{c}\text { Twenty Individual } \\
\text { 65\% HPGe detectors }\end{array}$ \\
\hline \hline${ }^{235} \mathrm{U}, 185.72 \mathrm{keV}$ & $47 \mathrm{~g}, 100 \mu \mathrm{Ci}$ & $29 \mathrm{~g}, 62 \mu \mathrm{Ci}$ \\
${ }^{239} \mathrm{Pu}, 375.92 \mathrm{keV}$ & $0.8 \mathrm{~g}, 48 \mathrm{mCi}$ & $0.2 \mathrm{~g}, 14 \mathrm{mCi}$ \\
${ }^{241} \mathrm{Am}, 59.35 \mathrm{keV}$ & $100 \mu \mathrm{Ci}$ & $44 \mu \mathrm{Ci}$ \\
${ }^{137} \mathrm{Cs}, 661.65 \mathrm{keV}$ & $90 \mu \mathrm{Ci}$ & $28 \mu \mathrm{Ci}$ \\
* The NaI MDA data were obtained from the MDA.EXE program written by \\
R. Maurer of the Washington Aerial Measurements Operation (WAMO).
\end{tabular}

Note that multiple scattering in air has not been included in the calculated $\mathrm{NaI}$ data, but will not be a significant problem at the 50 foot altitude chosen. This type of scattering must be taken into account at altitudes of 400 to 500 feet above the source plane. In addition to this caveat, the 50-foot altitude may not be practical for most urban scenarios due to power line hazards, tall buildings, etc.

If the aircraft speed is increased the point source MDAs are increased since fewer source counts are acquired, but the background counting rate will remain constant. This increase is minimal if the ground 'footprint' of the detector array is transited in 1 second or more. At low altitudes, the diameter of this 'footprint' is taken to be twice the altitude, i.e., 100 feet in diameter at a 50-foot altitude. A 1-second transit time yields a speed of about 60 knots. Higher speeds than this increase the MDA.

If the point sources are not directly below the flight path, but offset laterally by 50 feet, the MDAs are increased. This is caused by an effective detector area decrease as the angle between the source and the detector moves away from directly below the detector, i.e, the side area of the detector is less than the face area. In addition, the source-to-detector distance is increased which decreases the number of gammas incident upon the detector due to $1 / \mathrm{r}^{2}$ and increased air attenuation. These factors increase the MDA by approximately six times at a 50 -foot offset. 


\section{DATA ANALYSIS}

\section{Near Real Time in the Air}

Searching for specific sources is possible in near real time if the on-board data-processing software operates quickly. This is a non-trivial problem due to the high data rates from arrays of gamma detectors. Typically a computer program samples a limited number of gamma energy regions to determine if a significant number of counts have occurred (the $3 \sigma$ decision level). This requires that all detector counts be summed (with various gain and offset corrections) over the nominal 1 second of collection time. This summed spectrum is background corrected (for $\mathrm{NaI}$ ) and each gamma energy region is examined to see if the peak found in it exceeds $3 \sigma$. The results (and probably the spectral energy region) must then be displayed so the equipment operator can judge the result and suggest flight plan changes to the pilot. In addition, the computer hardware must store all the raw data for potential post-flight analysis.

The continuing development of inexpensive high-speed computer hardware (processors, memory, etc.) can have a significant impact on the data handling techniques that could be used on newly constructed aerial detection systems. In particular, it will be possible to collect $\mathrm{NaI}$ data on an event by event basis similar to the method used for the HPGe systems described in this note. The resulting data stream could then be used to generate more specific guidance information for the aircraft pilot, perhaps reflecting counting rates from specific isotopes.

In addition, the event by event (or list mode) data will allow ground-based summation of counts from individual detectors versus time with gain and offset corrections included on a detector by detector basis. These individual detector data can then be assembled into spectra with better energy resolution than that expected from an average correction applied to all detectors. Lastly, the large hard discs currently available can be used for removable data storage in the aircraft. After a data collection flight the discs can then be physically transferred to the land-based analysis equipment (see the following section) where the large data files will be quickly offloaded for processing.

The NaI detectors produce significantly more data per unit than a comparable HPGe array because of the higher detection efficiency. The only effective way to utilize this data stream is by application of the best and fastest data transfer and storage techniques currently available. These techniques will lead to improvements in near-real-time processing in the aircraft, but the most benefit will occur in accelerated on-ground analysis due to the higher data transfer rate from stored information to the processing software.

\section{After-the-Fact Analysis on the Ground}

Data analysis on the ground can be made more robust than that done in the air. One reason is that the computing power can be significantly greater, and more time can be taken with complicated analysis procedures: for example, background subtraction for $\mathrm{NaI}$ spectra. Hard copy of the data results will also be possible. It may also be desirable to create backup files for future reference. In general both in-air and on-ground analyses are useful. 
Spectral data, navigation information (altitude, GPS coordinates, or other LAT/LON information), vibration sensor data, and perhaps some system parameters such as detector temperature and electronic voltages are collected during each flight. The amount of information collected is large, 600 Mbytes for 2 hours of HPGe array flight, for example; and data storage must accommodate this mass of data. Ordinarily this is an electronic problem, but rapid data analysis on the ground is directly dependent upon the rate with which these data can be accessed on the ground. It has been found that removable hard discs work well as storage media in this application. The usual 1 to 2 Gigabyte type mounted on vibration isolators are satisfactory. The advantage is that upon termination of a flight, the hard disc with flight data can be given to an analysis crew, a new disc installed in the aircraft, and another flight initiated. Hard discs are also considerably cheaper than most helical scan tape drives of comparable capacity and offer random access to any file.

The initial after-the-fact data analysis process is generally similar for both the NaI and HPGe array data. Data analysis must handle individual detector spectra collected during a flight by correcting for gain differences, and offsets in the amplifiers. Typically, all detectors are summed together after these corrections have been made. The time stamps, GPS values, and other navigational information from the flight are then associated with the spectra. The resulting spectra are then made available at 1-second intervals over the flight time. If the survey consisted of a series of parallel flight paths with $180^{\circ}$ turns at each end, the software must eliminate the data in these turns since, typically, system vibration noise increases and the altitude is significantly different during the turn. If a linear path is flown, following a road, for example, then all data are used.

The next steps of data analysis differ considerably between NaI and HPGe. Usually one is interested in the physical distribution of particular isotopes on the soil surface. The NaI spectra must have some form of background stripping applied to minimize the effects of the natural background. This can be automated somewhat but better results are usually obtained by a person trained in the task. After removal of the background data, the net counts should represent new sources. Experience is a valuable tool here. Obviously a good quality background spectrum must be available. The net data are then used for plotting.

The HPGe data may be analyzed for particular gamma peaks by selecting the appropriate energy band. Since the HPGe peaks are very narrow, it may be possible for software to examine the summed spectra and produce net count data versus geographical position with little or no personal intervention. A background spectrum may not be needed for this task, but correction for ${ }^{226} \mathrm{Ra}$ gammas at the ${ }^{235} \mathrm{U}$ energy may be made more accurate by careful selection of background spectra and the resulting peak ratios.

Data from a series of parallel flight lines can be converted into isopleths or contours of constant intensity for particular energy bands. This process is complicated but has the advantage that adjacent flight line data can be used to better delimit a source location. The isopleths can be displayed on a map and printed for future use. Some of this data presentation is very time consuming unless suitable (and costly) equipment is used.

Linear flight data can be displayed versus actual geographic path and plotted to overlay on a map. In this instance, signal levels can be represented by various size symbols, larger diameter for 
greater counting rates, for example, colors correlated with signal level, or, less usefully, by alphanumeric characters. 
This page intentionally left blank 


\section{OPERATIONAL CONSTRAINTS}

This section addresses various aspects of detector arrays and aircraft platforms that affect choice of a system for field use. The most important differences have been noted above: sensitivity and isotope ID capability. These are clearly foremost in the list of field characteristics. Other considerations may be nearly as important however.

\section{NaI Detector Array}

Large NaI arrays have been in use for several years both on fixed-wing aircraft (initially) and now on helicopters. The arrays, the pod, and the electronics are sufficiently light that a small helicopter like a BO-105 is satisfactory. There are some weight constraints, but all are manageable. There are temperature considerations however (see Field Reliability).

\section{LN-Cooled HPGe Array}

The HPGe detectors must be operated at low temperatures (near $90 \mathrm{~K}$ ) provided by some form of cryogen, either $\mathrm{LN}$ or a J-T cooler using argon gas. A LN cooled array will use 10 or more liters of LN every 4 hours during flight. Slightly less is used when the array is stationary. Cool down time is about 6 hours so in practice the array is kept cold continuously. LN can be stored in large Dewars for some time, but not much longer than a few weeks. Thus it may be necessary to provide $\mathrm{LN}$ from a base station on a routine basis.

An array of 20-HPGe detectors, divided into two groups of ten, each with its own Dewar, will weigh about 760 pounds including the pod, umbilical cable to the aircraft, vibration support structures, and some electronics. Detector control, data recording and storage will add more weight. Commercial electronics necessary for this array used to weigh 1,010 pounds bringing the total to nearly 1,800 pounds. This size load requires a large helicopter (Bell-412 for example). Recent custom electronics has dropped the weight of the electronics to about 45 pounds, but even with this improvement, a small helicopter (BO-105 for example) has a limited in-air time.

A small helicopter may be able to carry only one to 2 hours of fuel if a heavy detector array is used. In practice this will limit the flight time somewhat and lengthen the survey hours. Even large helicopters have a limited flying time. With 1,800 pounds of detector gear 4 hours was a common flying time for an $\mathrm{SH} 3$ type helicopter.

\section{J-T-Cooled HPGe Array}

Some of these HPGe array logistic and weight problems can be minimized by using a J-T cooling system in place of LN and its Dewar. The high pressure argon gas needed for this cooling method can be stored indefinitely and hence there is no need for ongoing delivery of cryogen to a forward area. The cool down time is still 6 hours, however, and in practice the arrays are kept cool continuously. The argon consumption rate is about 4 standard liters per minute per detector so a considerable amount of gas must be on hand. It is also possible to run these devices on high pressure air which can be compressed on site. This is a promising, but as yet untried method. 
This cooling method does not need a Dewar for the detectors and the resulting array is lighter and smaller than the LN cooled version. The helicopter must carry a fiberglass tank of argon however. The overall weight of the J-T cooled array, including all electronics, is about 450 pounds for an array of $20110 \%$ HPGe detectors. Each detector this size weighs 10 pounds including its J-T cooler. The argon gas tank and a full load of argon gas weighs 307 pounds bringing the total to 760 pounds. This still makes it marginal for the BO-105 size helicopter; flight times will be less than one hour. A Bell-412 size helicopter has few limitations.

\section{J-T-Cooled Array in a UAV}

A subset of the 20-detector array using J-T cooling may be useful on a UAV. In this application weight is very important and the J-T cooling method is necessary. A UAV will have the advantage of a better flight envelope (i.e., it can fly closer to the ground surface) since personnel safety would not be of concern. Reducing the altitude from 50 feet to 10 feet for a UAV would increase the overall counting rate by nearly a factor of 25 . This will result in a single detector sensitivity comparable to a larger array at the higher altitude. This is an unexplored area at present.

\section{Fixed-Wing Aircraft}

A fixed-wing aircraft cannot fly as slowly as a helicopter. In addition, a fixed-wing aircraft can only safely fly at 200 feet or greater. The effective ground footprint of the detector system will be about 400 feet in diameter at this altitude implying a speed of 240 knots for a 1 second transit across the footprint. This will theoretically allow collection of approximately the same number of gammas from the source as a 50 foot helicopter flight at 60 knots, but air attenuation and 1/ $r_{2}$ will reduce the actual number arriving at the detector. The result is a factor of approximately 30 increase in the MDA for ${ }^{235} \mathrm{U}$ compared to the 50 foot altitude.

If safety considerations require a higher aircraft speed (say 300 knots), then the MDA will be increased more since the shorter residence time over the source will reduce the total number of counts recorded.

A detection system mounted on a fixed wing aircraft will thus have poor point source sensitivity compared to the same system mounted on a helicopter flying at a lower speed. The result is a smaller peak that could be hidden in the noise.

\section{External Stores vs Internal Mounting of Detector Array}

Another constraint that must be considered is placement of the detector array. The highest counting rate is obtained with the least shielding (attenuation) between the detectors and the source. In addition, shielding always attenuates the lower energy gammas more than higher energy ones. This increases the MDA's for ${ }^{235} \mathrm{U}$ for example compared to ${ }^{137} \mathrm{Cs}$.

If the detector array is mounted as an external store on an aircraft the shielding will probably be a minimum. However the pod, its construction, and its mounting attachment to the aircraft must be sufficiently rugged (i.e., a better shield) so that it will not fail and become a hazard to aircraft 
operation. This is part of the airworthiness evaluation that must be done on any external store. This may be a lengthy and costly process.

If the detector array is mounted internally then some of the safety concerns and rugged construction of the array housing are minimized, but now the aircraft structure itself may provide shielding between the detectors and sources on the ground. Some helicopters, for example, have fuel tanks under the floor. This is a significant attenuator, but, worse, the attenuation varies with flight time as the fuel is used up. 
This page intentionally left blank 


\section{FIELD RELIABILITY}

Experience has shown that either of the two systems noted above, NaI or HPGe, can be operated reliably in the field. There has been considerably more field time with the NaI systems, and most of the obvious bugs have been worked out. Each system will have unexpected failures. Hence field servicing is an important consideration.

\section{NaI Detector Array}

$\mathrm{NaI}$ scintillators are affected by sudden changes in temperature. The result of a rapid change in temperature is cracking of the scintillator crystal leading to complete failure of the detector. Probably climatic changes are not rapid enough, but moving from a warm hanger to $-20^{\circ} \mathrm{C}$ outside is a significant problem. Effects of these temperature changes may be minimized by insulating the scintillators to limit the rate of change of crystal temperature. The HPGe detectors have no problems of this nature.

The NaI scintillators and the HPGe detectors are fragile, but the usual packaging will prevent most accidents (and will serve as thermal insulation for the NaI scintillators).

NaI scintillators have an additional temperature problem: their gain drifts with temperature. The practical import of this is a necessity for frequent calibration or manual gain adjustment. If this is not part of the operating protocol the data analysis is complicated and may even fail.

\section{HPGe Detector Array}

The LN-cooled HPGe system must be filled with cryogen every 4 hours or so. This has been a source of difficulty in the past. Most field personnel are not familiar with cryogen handling and careful operator training is required. Automatic filling systems have not proven to be reliable enough.

The J-T-cooled HPGe detectors will need high pressure gas (argon) for operation. High pressure gas handling is fairly routine since many aircraft power booster systems (landing gear for example) use high pressure air or nitrogen. Thus it is not expected that the argon gas handling is as problematical as $\mathrm{LN}$ handling has been, however if the argon is in standard gas bottles they are large, heavy, and awkward.

\section{Detector Servicing}

In the event of a failure replacement of a detector is a likely scenario. The NaI systems are very easy to work with in this case. Simple detector swap out is a few minutes followed by recalibration.

The HPGe detector systems are much more of a problem. For example if a detector becomes noisy the software may be able to switch its data stream off with little problem to data collection. However if an LN-cooled detector suffers a vacuum failure the whole Dewar can be taken down losing significant sensitivity. In this case it would be prudent to remove and replace the failed 
detector. This is a two day job since the array must be brought up to room temperature before the offending detector can be removed, and then the actual replacement will take a few hours followed by a 6 hour cool down.

The J-T-cooled HPGe array has individual detectors that are independent of one another. If one detector develops a leak and starts to use too much argon it can be removed from the system fairly easily. It is difficult to replace a detector since the whole array would probably need to be warmed up and re-cooled with about the same difficulty as for the LN cooled system.

Field service of the electronics is nearly identical for either system. 


\section{COST AND AVAILABILITY}

The NaI detectors are readily available with many international vendors. Even the large $2 \mathrm{x} 4 \mathrm{x}$ 16-inch scintillators at $\$ 6,000$ each cost much less than any HPGe detector. Delivery is fairly rapid. A NaI system can be assembled by the user with little difficulty. Custom electronics has to be designed, constructed, and tested since none is available commercially. Estimation of costs for custom electronics is difficult, but a recent design with modern electronics will probably cost $\$ 300,000$. If a small helicopter is to be used then special consideration must be given to weight both in the scintillator mounting techniques and in the electronics design and packaging. This complicates the whole design process considerably.

The LN-cooled HPGe detectors are much more costly than the NaI scintillators and are available from many international vendors. A $65 \% \mathrm{LN}$-cooled detector is about $\$ 40,000$. If a complicated Dewar is needed for multiple detectors this will usually have to be designed, fabricated, and tested by the detector vendor. Cost of a ten-detector Dewar will be about $\$ 40,000$ and will be a special purpose design. The special design may make ad hoc changes (to fit a different aircraft for example) very difficult.

The J-T-cooled HPGe is at present available from only one vendor, but a large array has been made out of these detectors (20-110\% detectors) for helicopter-borne applications. The $110 \%$ $\mathrm{J}-\mathrm{T}$ detectors are about $\$ 80,000$ each. No Dewar is needed, but a gas handling system will be required for the high pressure argon gas. This will cost about $\$ 75,000$ including a fiberglass tank on board the aircraft.

Both versions of HPGe detectors require custom electronics to meet size, weight, and data transfer requirements. Existing custom electronics are VME-based and can be made fairly small, but the design is unique for each application. The electronics cost will be slightly more than the $\mathrm{NaI}$ version, perhaps $\$ 350,000$, since most of the functions will be the same but more ADCs will be needed.

The software for control and data collection have been factored into the electronics cost.

Analysis software will also be custom, and specific to the application. Software for this purpose is not complicated and costs of $\$ 150,000$ are to be expected. 
This page intentionally left blank 


\section{FIELD DETECTION DISCUSSION}

\section{A Large Search Area Requires a Long Time}

Use of NaI and HPGe detector arrays (perhaps supplemented by neutron detector arrays) for locating and identifying radiation sources in a field scenario is not foolproof. Searching a large area takes a long time because the detector must be within a few 10's of feet (a 50 foot altitude was used above) of the source to positively locate and subsequently identify the isotope. If the supposed source location is totally unknown the searching routine will take a long time. An estimate of actual time-in-the-air needed to search a square mile at a 50 foot altitude with 75 foot line spacing is about four hours (not including transit time to the location). If intelligence means can be used to limit the search area a detector system still must be brought close (50 to 100 feet) to the source for identification to be successful.

\section{Deliberate Shielding of Sources}

A knowledgeable person can shield most of the SNM source gammas with little effort. In many cases a few cm of lead will make detection very difficult. Sources buried in soil, sand, or in underground structures may never been seen with gamma detectors. Sources viewed off-axis can accidentally be shielded by uneven terrain.

In some cases a neutron detector can be used more effectively than a gamma detector, and is the preferred detector for a few SNM scenarios. Shielding of neutrons is more difficult than gammas and may even be neglected by the source owner since common instruments are not sensitive to neutrons and their presence may not be appreciated. See the following section on Array Variations.

\section{Identification of Sources and Source Producing Operations}

The detector systems discussed in this note, HPGe arrays in particular, can be used to identify radioactive sources by isotope. This information is valuable and is necessary to evaluate the threat presented by the source presence.

The same type of data can also be used to characterize an industrial process by verifying the production, or use of radioactive material. In these cases the more definitive the data the better. For example if short half life isotopes are found, some means of production must be nearby, possibly a reactor or accelerator. The presence of fission products indicate fuel reprocessing and potential SNM production. Contamination along roads can lead to source facilities.

Fuel reprocessing can release radioactive gas such as ${ }^{85} \mathrm{Kr}$ that can be identified by its gamma signature. In many countries this gas is captured and not released due to safety concerns. It is possible that some clandestine operations might not be so careful and identification of this isotope may reveal useful details. Gaseous isotopes are of course readily diluted by mixing with the environmental air so that concentration $\left(\mathrm{Ci} / \mathrm{m}^{3}\right)$ may be very low. This is a difficult detection problem. 
Some construction tasks produce unlikely concentrations of naturally occurring radioactive materials. Excavation of an underground structure, for example, may result in locally deposited 'spoil' that may be revealed by a radioactive signature that differs from the immediate surroundings. Some drilling tailings or mud is more radioactive than the surrounding dump site. In each of these cases high resolution gamma spectra can be used to verify the difference, and analysis may be able to suggest the origin of the foreign material. 


\begin{abstract}
ARRAY VARIATIONS
Field experience has shown that each of the detector systems described above has some advantages that the other does not. NaI sensitivity and HPGe resolution are obvious specific differences. It may be possible to combine detector types and use each detector to better advantage by employing a more narrowly defined detection scheme.
\end{abstract}

\title{
Combined NaI and HPGe Arrays
}

An example of this is use of a large $\mathrm{NaI}$ array with its high sensitivity to find a gamma anomaly rapidly. This anomaly is then marked for subsequent isotope identification using an HPGe array. One simple way this might be done would be to use a helicopter-borne $\mathrm{NaI}$ array to find a source then land or hover over the anomaly with an extended collection time to permit a smaller HPGe array to acquire enough data to permit proper analysis. Obviously this is scenario dependant; perhaps the location being surveyed will not permit either a hover or a landing. In this case multiple passes over the anomaly may work.

This type of interchange between detector systems is dependent upon anomaly detection in near real-time (on board the helicopter) for best performance. This implies that the system software is capable of providing this kind of information using some form of quick analysis. Perhaps something as simple as a rate meter sensing gross counting rate, or the rate in a broad window that excluded as much background as possible, would suffice. It may be possible to mark the anomaly with a GPS location and revisit the location later with a different detection system (although this sounds clumsy).

Multiple detector types used together may have beneficial cost tradeoffs, but the downside is maintenance of two separate and different detection systems. If the actual scenario permits, one variation that seems satisfactory is a large $\mathrm{NaI}$ array accompanied by a hand-held HPGe detector that can be brought very close to the anomaly upon landing. Access via land vehicle may also be considered. When a source is found and is highly radioactive then a large HPGe detector may need only a few hundred seconds of data collection to make an accurate isotopic identification.

\section{Different Vehicular Platforms}

For some search scenarios such as built up areas in towns and cities, aerial platforms may be very difficult to use. Both NaI and HPGe systems have been successfully deployed in vans, trucks, and railway cars. The logistics are similar to the aerial problems noted above, but the safety aspects and searcher profile are greatly simplified.

Detector sensitivity is usually better for van and truck based detectors than for aerial platforms since they will be closer to the sources. Background sources are also closer, and the natural variability may be a problem.

\section{Neutron Detectors Combined with Gamma Detectors}


SNM which contain plutonium emit neutrons. Detection of these neutrons using large arrays $(2 \mathrm{x}$ $4 \times 8$ feet) of ${ }^{3} \mathrm{He}$ proportional counters in polyethylene moderator is quite practical. This follows from the low natural neutron background and the small variation in this background versus geographical location. This leads to small values of $3 \sigma$ for neutrons which in practical terms relates to a high probability of detection with a low probability of false alarms. Any significant increase in neutron fluence rate is caused by man-made sources or structures.

A large neutron detector array is light weight and may be combined with aerial or ground vehicle mounted gamma detectors.

It is more difficult to shield neutrons, and shielding may even be ignored by some workers, so neutron detection is of primary concern when searching for some types of plutonium containing sources.

\section{UAV Applications}

Another variation of the standard detection schemes is a single large $110 \% \mathrm{~J}$-T cooled HPGe detector on a UAV. As discussed previously, this has the advantage that a UAV can be flown closer to the ground than any manned aircraft and will be closer to the source. The increase in counting rate between 10 feet and 50 feet is about a factor of 20. Thus the array MDAs presented in Table 1 will approximately apply to the single detector. 


\section{CONCLUSIONS}

The salient conclusions are briefly listed in Table 3 below.

Table 3. Aerial gamma detection systems comparison.

\begin{tabular}{|c|c|c|c|}
\hline System Characteristic & $\begin{array}{c}\text { Eight Individual } \\
2 \times 4 \times 16-i n c h \\
\text { NaI Scintillator Logs } \\
\end{array}$ & $\begin{array}{c}\text { Twenty Individual } \\
\text { LN Cooled } \\
\text { 65\% HPGe Detectors } \\
\end{array}$ & $\begin{array}{c}\text { Twenty Individual } \\
\text { J-T Cooled } \\
\text { 110\% HPGe Detectors } \\
\end{array}$ \\
\hline $\begin{array}{l}\text { Point source sensitivity, } \\
3 \sigma \mathrm{MDA} \\
\begin{array}{l}{ }^{233} \mathrm{U} \\
{ }^{239} \mathrm{Pu} \\
{ }^{241} \mathrm{Am} \\
{ }^{137} \mathrm{Cs}\end{array} \\
\end{array}$ & $\begin{array}{c}47 \mathrm{~g}, 100 \mu \mathrm{Ci} \\
0.8 \mathrm{~g}, 48 \mathrm{mCi} \\
100 \mu \mathrm{Ci} \\
90 \mu \mathrm{Ci} \\
\end{array}$ & $\begin{array}{c}29 \mathrm{~g}, 62 \mu \mathrm{Ci} \\
0.2 \mathrm{~g}, 14 \mathrm{mCi} \\
44 \mu \mathrm{Ci} \\
28 \mu \mathrm{Ci} \\
\end{array}$ & $\begin{array}{c}17 \mathrm{~g}, 37 \mu \mathrm{Ci} \\
0.1 \mathrm{~g}, 8 \mathrm{mCi} \\
26 \mu \mathrm{Ci} \\
17 \mu \\
\end{array}$ \\
\hline Peak counting rate & 1.0 & 0.1 & 0.2 \\
\hline $\begin{array}{l}\text { Energy resolution at } \\
186 \mathrm{keV}\end{array}$ & $10 \%$ & $1 \%$ & $1 \%$ \\
\hline Isotope ID & very difficult & easy & easy \\
\hline Data rates & low & high & high \\
\hline System weight & low & high (for old design) & medium \\
\hline Field support & simple & $\begin{array}{l}\text { high complexity, fill } \\
\text { with LN every } 4 \text { hours }\end{array}$ & $\begin{array}{l}\text { medium complexity, fill } \\
\text { with high pressure argon } \\
\text { gas every flight }\end{array}$ \\
\hline Airworthiness & proven & proven & proven \\
\hline Fragile? & $\begin{array}{l}\text { yes, rapid temperature } \\
\text { changes a problem }\end{array}$ & $\begin{array}{l}\text { no, but mechanical } \\
\text { complexity, LN filling a } \\
\text { problem }\end{array}$ & $\begin{array}{l}\text { no, but cooling gas purity } \\
\text { must be maintained }\end{array}$ \\
\hline $\begin{array}{l}\text { Field servicing of bad } \\
\text { detector }\end{array}$ & very easy & very complicated & moderately complicated \\
\hline Support electronics & $\begin{array}{l}\text { light weight, fairly } \\
\text { complex }\end{array}$ & $\begin{array}{l}\text { heavy, complicated (due } \\
\text { to old designs) }\end{array}$ & light, complicated \\
\hline Cost & low $-\$ 350 \mathrm{~K}$ & high - $\$ 1.3 \mathrm{M}$ & high $-\$ 2.1 \mathrm{M}$ \\
\hline Availability & $\begin{array}{l}\text { readily available, many } \\
\text { vendors }\end{array}$ & $\begin{array}{l}\text { detectors readily avail- } \\
\text { able, but Dewars are } \\
\text { custom, many vendors }\end{array}$ & $\begin{array}{l}\text { detectors available, one } \\
\text { vendor at present, array is } \\
\text { custom constructed by } \\
\text { user }\end{array}$ \\
\hline $\begin{array}{l}\text { Adaptability to different } \\
\text { aircraft }\end{array}$ & $\begin{array}{l}\text { yes, needs external } \\
\text { ordnance hangers }\end{array}$ & $\begin{array}{l}\text { system components are } \\
\text { large, may not fit all } \\
\text { helicopters, used on } \\
\text { external ordnance } \\
\text { hangers }\end{array}$ & $\begin{array}{l}\text { detector pod fairly large, } \\
\text { needs external or internal } \\
\text { mounting, electronics are } \\
\text { small }\end{array}$ \\
\hline
\end{tabular}


